\title{
Radiographic and histologic effects of bone morphogenetic protein-2/hydroxyapatite within bioabsorbable magnesium screws in a rabbit model
}

Le Hoang Nam Dang ${ }^{1 \dagger}$, Yu Kyoung Kim²${ }^{2}$ Seo Young Kim², Kuk Jin Lim', Ken Bode ${ }^{1}$, Min Ho Lee ${ }^{2}$ and Kwang Bok Lee ${ }^{1 * \dagger}$

\begin{abstract}
Background: Hydrogen gas formed by magnesium (Mg) screw corrosion can accumulate around the implant and create bone cysts, long-term osteolysis lesions, and bone healing delay. Thus, several authors currently do not recommend Mg implants for clinical use. In contrast, bone morphogenetic proteins (BMP)-2 have a very strong osteoinductive activity. The purpose of this study was to evaluate the effect of rhBMP-2/hydroxyapatite (HA) inside specially designed Mg cannulated screws in a rabbit femur model for hydrogen gas formation avoidance.

Methods: Fifteen rabbits underwent randomly different cannulated Mg screw implantation in both distal femora; 30 femora were divided into three groups depending on the materials fill in the cannulated $\mathrm{Mg}$ screw: control group (Mg screw with no treatment), HA group (Mg screw with HA), and BMP-2/HA group (Mg screw with a composite BMP-2/HA). Plain radiography, micro- $\mathrm{CT}$, and histological analysis were accomplished, and the ability to release BMP-2 of the screws was evaluated by immersion of both the screw with no treatment and screw with a composite BMP-2/HA into the SBF for up to 7 days.

Results: X-ray assessment found the gas shadow around the implant was slightly smaller in the BMP-2/HA group than the HA and control groups at 8 weeks. Micro-CT analysis demonstrated statistically significant higher new bone formation in the BMP-2/HA group than the other groups, respectively, which also correlated with a decreased gas volume. Histological analysis showed higher osteointegration between implants and host femurs in the BMP-2/HA group than the HA and control groups at 12 weeks.
\end{abstract}

Conclusions: This study indicates that the combination of BMP-2/HA within Mg screws enhances new bone formation and therefore has the potential to decrease the complications of hydrogen gas formation around these implants.

Keywords: Magnesium screw, Bone morphogenetic protein 2, Hydrogen gas, Hydroxyapatite

\section{Background}

Biodegradable metals are promising options in solving the dilemma of stress shielding and secondary surgical interventions. Among these types of biodegradable metals, $\mathrm{Mg}$

\footnotetext{
*Correspondence: osdr2815@naver.com

'Le Hoang Nam Dang and Kwang Bok Lee contributed equally to this work. 'Department of Orthopedic Surgery, Chonbuk National University Medical School, Research Institute of Clinical Medicine of Chonbuk National University-Biomedical Research Institute of Chonbuk National University Hospital, Jeonju, South Korea

Full list of author information is available at the end of the article
}

has been widely and systematically investigated for biomedical applications. The rigidity of $\mathrm{Mg}$ is greater than ceramic biomaterials, and the elastic modulus and compressive yield strength of $\mathrm{Mg}$ are closer to those of natural bone than other generally used metallic implants [1]. Because of their similar elastic moduli, using Mg implants in boney applications would greatly reduce the implant's stress shielding that could inhibit bone resorption. Furthermore, $\mathrm{Mg}$ ions are an essential element of the human organism, as the fourth most abundant cations in the human body, with approximately half of the total Mg stored 
in the bone tissue [2]. In addition, several authors report $\mathrm{Mg}$ to have good biocompatibility and may have stimulatory effects on the new bone formation. These properties assist the material's consolidation into the surrounding bone and possibly allow full regeneration after degradation $[1,3,4]$.

Unfortunately, the main disadvantage of $\mathrm{Mg}$ is a high corrosion rate with resultant hydrogen gas formation on contact with fluids [5]. This gas can induce complications such as superficial skin necrosis, and the gas bubbles can accumulate around the implant creating complications of bone cysts and long-term osteolysis lesions. These lesions can seriously delay the bone healing so that several authors are currently not recommending their clinical use [6-8]. In order to overcome this limitation of $\mathrm{Mg}$ implants, many techniques have been employed such as alloying, protective coatings, and surface treatments [9, 10]. The coating method was effective in diminishing the size of the voids in the surrounding bone but did not fully prevent degradation, although it was effective in avoiding the undesired initial burst release of gas [11]. Therefore, it is necessary to attempt different solutions to overcome these complications relative to hydrogen gas formation.

Bone morphogenetic proteins are members of the transforming growth factor-beta superfamily. Multiple bone morphogenetic proteins (BMP) are considered to have a crucial signaling role in chemotactic proliferation and differentiation of osteoprogenitor cells, thereby inducing bone formation [12]. Among the BMP, BMP-2 is the most extensively researched and has very strong osteoinductive activity [13]. Hydroxyapatite (HA) is used as a bone graft extender for posterolateral spinal fusion in humans [14]. It is also beneficial as a BMP-2 carrier because of its high attraction for BMP-2; BMP-2-adsorbed hydroxyapatite is a safe promoter of bone formation [15].

For the above reasons, this study used specially designed Mg cannulated screws with a BMP-2/HA delivery system inside the implants to evaluate the radiographic and histologic effects of this combination within a rabbit femoral model for avoiding hydrogen gas formation.

\section{Methods}

\section{Experimental design}

The selection of experimental animals, their management, and the surgical protocols were authorized by the Institutional Animal Care and Use Committee of the Chonbuk National University Laboratory Animal Center, Jeonju, South Korea (approved number: CBNU 2017-0013). Fifteen male New Zealand white rabbits (age, 2 months; body weight, $1.8-2 \mathrm{~kg}$ ) were used, and the 30 distal femora were divided into three groups of 10 which had different Mg screw preparations randomly implanted into both distal femora: control group (Mg screw with no treatment), HA group (Mg screw with HA), and BMP-2/HA group
(Mg screw with a composite BMP-2/HA). Four distal femora which contain samples from each group were collected at 8 weeks for micro-computed tomography $(\mu \mathrm{CT})$ and histological assessment. The remaining nine rabbits (six samples from each group) were assessed with plain radiographs at 1, 2, 4, 8, 12 weeks and sacrificed at 12 weeks for $\mu \mathrm{CT}$ and histological assessment.

\section{Implant preparation}

$\mathrm{Mg}$ alloy (Mg-Ca) cannulated screws were manufactured by U\&I company, South Korea. These were headless screws: $2.7 \times 10 \mathrm{~mm}$, cannulated hole diameter $0.9 \mathrm{~mm}$. Two $0.6 \mathrm{~mm}$ holes were placed in the body of the screw at a distance of $2.2 \mathrm{~mm}$ and $3.7 \mathrm{~mm}$ from the end of the screw, which connected the cannulated portion to the local environment and enabled elution of BMP from this section (Fig. 1a).

The BMP-2 used in this study was provided by Daewoong Pharmaceutical Co., Ltd. (Novosis ${ }^{\circ}$-dent, Gyeonggi, South Korea). Lyophilized BMP-2 was dissolved in $10 \mathrm{~cm}^{3}$ of distilled water to yield a concentration of 0.1 $\mathrm{mg} / \mathrm{ml}$. The doses of BMP-2 in this study were established based on the weight equivalent to the baseline dose that would be used in human undergoing spine fusion. The mean body mass, dose, and dose/weight assumed to humans were $70 \mathrm{~kg}, 1.5 \mathrm{mg}$, and $0.0214 \mathrm{mg} / \mathrm{kg}$, respectively, resulting in values for the rabbits of around $2 \mathrm{~kg}$, and the dose was $0.04 \mathrm{mg}$.

Hydroxyapatite, manufactured by Sigma-Aldrich company. (Sigma-Aldrich Korea 698-84 Maeng-ri, Wonsammyun Cheoin-gu, Yongin City 17166 South Korea), was used as the carrier material, and 20-mg HA was placed into the screw canals in the HA and BMP-2/HA groups. The exact amount of HA was calculated by the difference in screw weight before and after fill-in of HA into the canal.

\section{Animal model}

The animals were housed in separated cages under standard laboratory conditions and fed a standard diet. Rabbits were anesthetized with an intramuscular injection of a 3:1 solution of ketamine hydrochloride (Ketalar, Yuhan, Seoul, Korea) and xylazine (Rompun, Bayer Korea, Seoul, Korea). The lateral distal femurs were shaved and disinfected with iodine, and then infiltration anesthesia was induced by injection of $2 \%$ lidocaine (lidocaine- $\mathrm{HCl}$, Hans, Seoul, Korea).

The lateral distal femoral metaphysis was surgically exposed by incisions through the skin and fascia lata. The periosteum was then elevated by performing a full-layer incision with an \#11-blade, and using a periosteal elevator, the periosteum was gently dissected from the bone. The implant placement was $1 \mathrm{~cm}$ above the knee joint line on the lateral femoral condyle. The implants were 


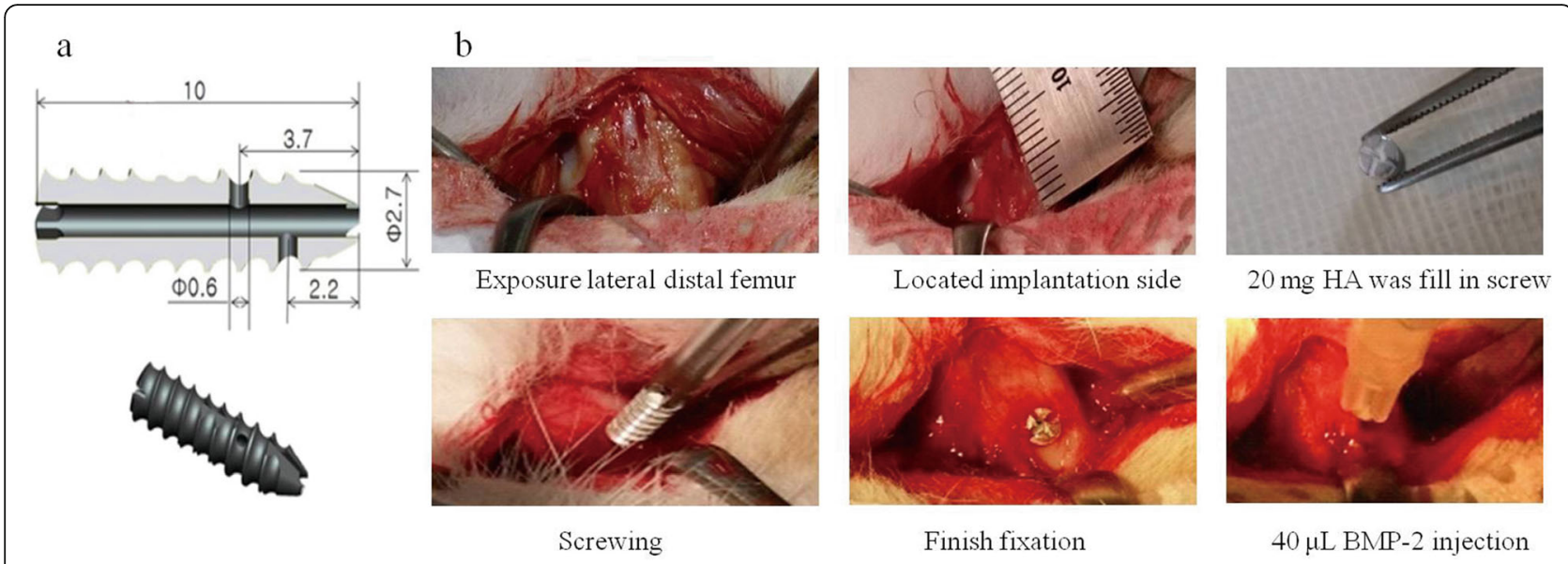

Fig. 1 Experimental design. a Mg cannulated screw with special design. b Different portions of the surgical procedure

manually screwed after drilling a hole of $2.5-\mathrm{mm}$ diameter and 10-mm depth using a low-speed drill and saline irrigation. The HA and BMP-2/HA were then placed inside the cannulated screws for those groups. The soft tissue was repositioned and then layer closured using 4-0 synthetic absorbable multifilament suture materials (VicrylPlus Antibacterial, Ethicon, Somerville, NJ, USA) (Fig. 1b).

Postoperative antibiotics (Amikacin; $1.5 \mathrm{mg} / \mathrm{kg}$ body weight) were administered by daily intramuscular injection for 1 week. During the postoperative stage, the animals were allowed to move freely in their cages without external support. Skin sutures were removed at 10 days postoperative.

\section{X-ray radiographs and micro-computed tomography analysis} Anteroposterior and medial-lateral view X-rays of the rabbit's treatment leg were collected to assess gas bubble (radiolucent/dark color) and bone formation (radiopacity/ gray color).

The bone specimens including screws were harvested by transaxial cutting $0.5 \mathrm{~cm}$ above and below the screw position. Block biopsy specimens were quantitatively analyzed using micro-CT (Skyscan 1076) at $100 \mathrm{kV}$ and $100 \mu \mathrm{A}$, respectively, with $240 \mathrm{~ms}$ of exposure time. The specimens were immersed in 10\% neutral buffer formalin solution during the time of imaging. The bone and gas formation volumes were estimated by the phantom in Hounsfield Units (HU) using CTAn software (Skyscan). The image from micro-CT scan was reconstructed by DataViewer software and reconstructed images were continued to be analyzed with CTAn software; the area to be analyzed from the reconstructed images for trabecular is specified by the region of interest (ROI), which is typically selected adjacent to the around screw and endocortical surface for trabecular bone. ROI selection is achieved using a freehand drawing tool, and auto-interpolation between the different ROI levels produces the total volume of interest (VOI) for all frames selected.

Gas volume was quantified on the same procedure with bone volume, but different in bone mineral density calibration value.

\section{Histological analysis}

After completing micro-CT scanning, the fixed distal femur blocks were immersed in Villanueva solution (Polysciences) for 3 days and dehydrated in a graded series of ethanols and acetone $100 \%$, and then embedded in methyl methacrylate resin. Transaxial sections (approx. 0.5 $\mathrm{mm}$ thickness) were cut, ground to $70 \mu \mathrm{m}$ thickness, and polished for hard tissue evaluation by optical microscopy (EZ4D, Leica).

\section{The ability to release BMP- 2 of the screw analysis}

To evaluate the ability to release BMP-2 of the screws, the screw with no treatment and screw within $40 \mu \mathrm{g}$ BMP-2/ $20 \mathrm{mg}$ HA were incubated in $1.5 \mathrm{ml}$ micro-tube containing $1 \mathrm{ml}$ of simulated body fluid (SBF, pH 7.4), under $100 \mathrm{rpm}$ at $37^{\circ} \mathrm{C}$. At predetermined time intervals of $1,2,3,6,12 \mathrm{~h}$ and $1,2,4,7$ days, the supernatant was completely removed and replaced with a fresh SBF solution. Samples were stored at $-20^{\circ} \mathrm{C}$ until the test. The amount of released protein was measured using the Human BMP-2 enzyme-linked immunosorbent assay (ELISA) kit. The absorbance of specimens was measured at a wavelength of $450 \mathrm{~nm}$ with ELISA reader (Model Spectra MAX PLUS, Molecular Devices, Sunnyvale, CA). The experiments were run in triplicate per time point.

\section{Statistical analysis}

The mean values and standard deviations for new bone formation and gas formation around screws data were calculated for each group. Shapiro-Wilk test was performed to test data for normal distribution. An 


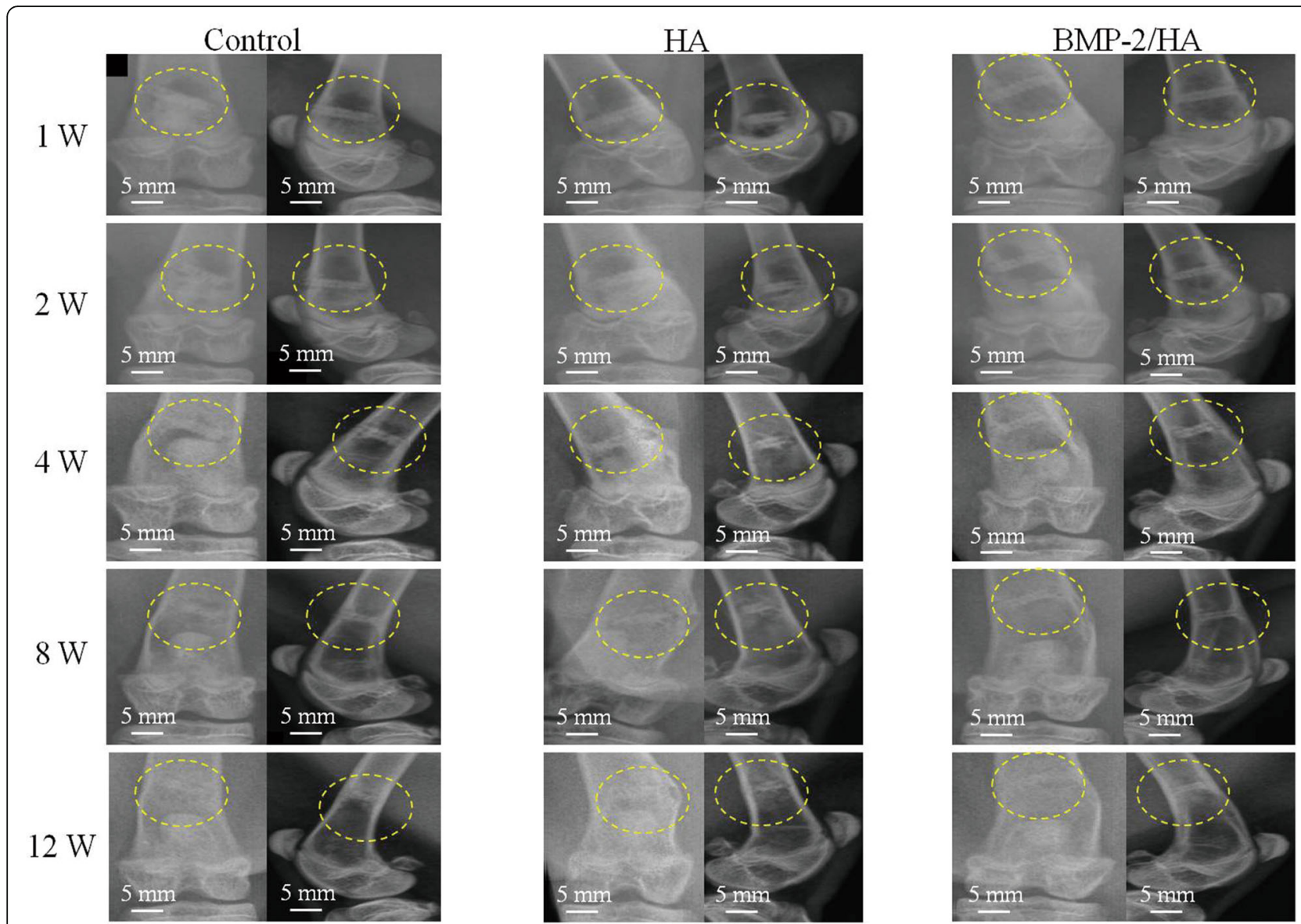

Fig. 2 Anteroposterior and lateral view radiographic images of the experimental region. Images were taken from 1 to 12 weeks postoperative. The yellow circles indicate the position of the screw

independent two-sample $t$ test (when the data had normal distribution) and Mann-Whitney $U$ test (when data had non-normal distribution) were conducted to compare differences between the groups. All tests were performed using SPSS software, version 20.0 (SPSS, Chicago, IL, USA), and $p$ values under 0.05 were considered statistically significant.

\section{Results}

All 15 rabbits survived the surgical procedure, and there were no wound infections, local inflammation, or morbidity in any of the animals.

\section{Radiographic evaluation}

Figure 2 comprises radiographs showing the postoperative change in each group; the yellow circles indicate the position of the screw. No evidence of implant migration was observed on radiographic evaluation up to 12 weeks.

In all animals, early gas formation around the screws was evident by 1 week postoperative (radiolucent areas around screw), and the gas expansion areas were larger after 2 weeks. At the 4-week time point, newly formed bone appeared (radiopacity areas), which sequentially replaced the gas around the implant. Of note, at 8 weeks, the radiopacity areas around the implant were slightly more in the BMP-2/HA group than the HA and control groups. This difference was especially evident by 12 weeks, as the control and HA groups had continued gas presence while the BMP-2/HA group had an apparent complete replacement of gas with newly formed bone. Likewise, the radiographic density appeared to be greater in the control/HA groups than in the BMP-2/HA group, respectively.

\section{Micro-CT evaluation}

In accordance with the radiographic findings, the coronal and sagittal plane micro-computed tomography images with red lines which indicate the position of the sagittal plane from the coronal plane of the screw, at 8 weeks and 12 weeks (Fig. 3), demonstrated the quality and quantity of new bone formation around the implant. The BMP-2/HA group showed a higher density of bone than the other two groups with bone formation more approximated to the implant surface in the BMP-2/HA group, respectively. 
At 8 weeks, similar new bone formation results were seen between the three groups, with higher bone volumes in the BMP-2/HA group than in the control/HA groups, but this difference was not statistically significant (Fig. 4a).

At 12 weeks, the micro-CT analysis demonstrated a significantly higher new bone volume formation in the BMP-2/HA and HA groups when compared to the control group $(p<0.001$ and $p<0.01$, respectively). The newly formed bone volume was also significantly higher in the BMP-2/HA group compared with the HA group $(p<0.01)$ (Fig. 4a).

Furthermore, according to the micro-CT analysis at 8 and 12 weeks, the volume of gas formed was significantly lower in the BMP-2/HA group compared with the other groups $(p<0.001)$, respectively. Also, the volume of gas formed around implants decreased from 8 to 12 weeks in each group (Fig. 4b).

\section{Histological evaluation}

At 8 weeks postoperative, histological analysis showed an implant and new bone interface in all groups; red arrows indicate newly formed osteocyte. However, the integration between implant and new bone within the
BMP-2/HA group was stronger than the HA and control groups, as the newly formed bone in the HA and control groups was scattered along the screw surface (Fig. 5a).

At 12 weeks postoperative, increased new bone formation was observed around implants in all groups. However, at the 8- and 12-week time points, the BMP-2/HA group showed stronger integration between implant and new bone when compared with the other groups. With yellow arrows indicating the gap between new bone layers and implant surface, small gaps were observed between the implant surface and newly formed bone in the HA group which was larger in the control group (Fig. 5b).

\section{The ability to release BMP-2 of the screw evaluation}

Figure 6 shows results demonstrating the measured quantities of BMP-2 released with time between the screw with no treatment and screw with treatment within $40 \mu \mathrm{g}$ BMP-2/20 mg HA. During 7 days, the concentration of released BMP-2 in the screw treatment within BMP/HA was above $8032.593 \pm 1900.805 \mathrm{pg} / \mathrm{ml}$, and the results revealed a rapid increase in BMP release during the first $1 \mathrm{~h}$ and release profile continued to increase over the 7-day period; the total amount of BMP-2 released after 7 days

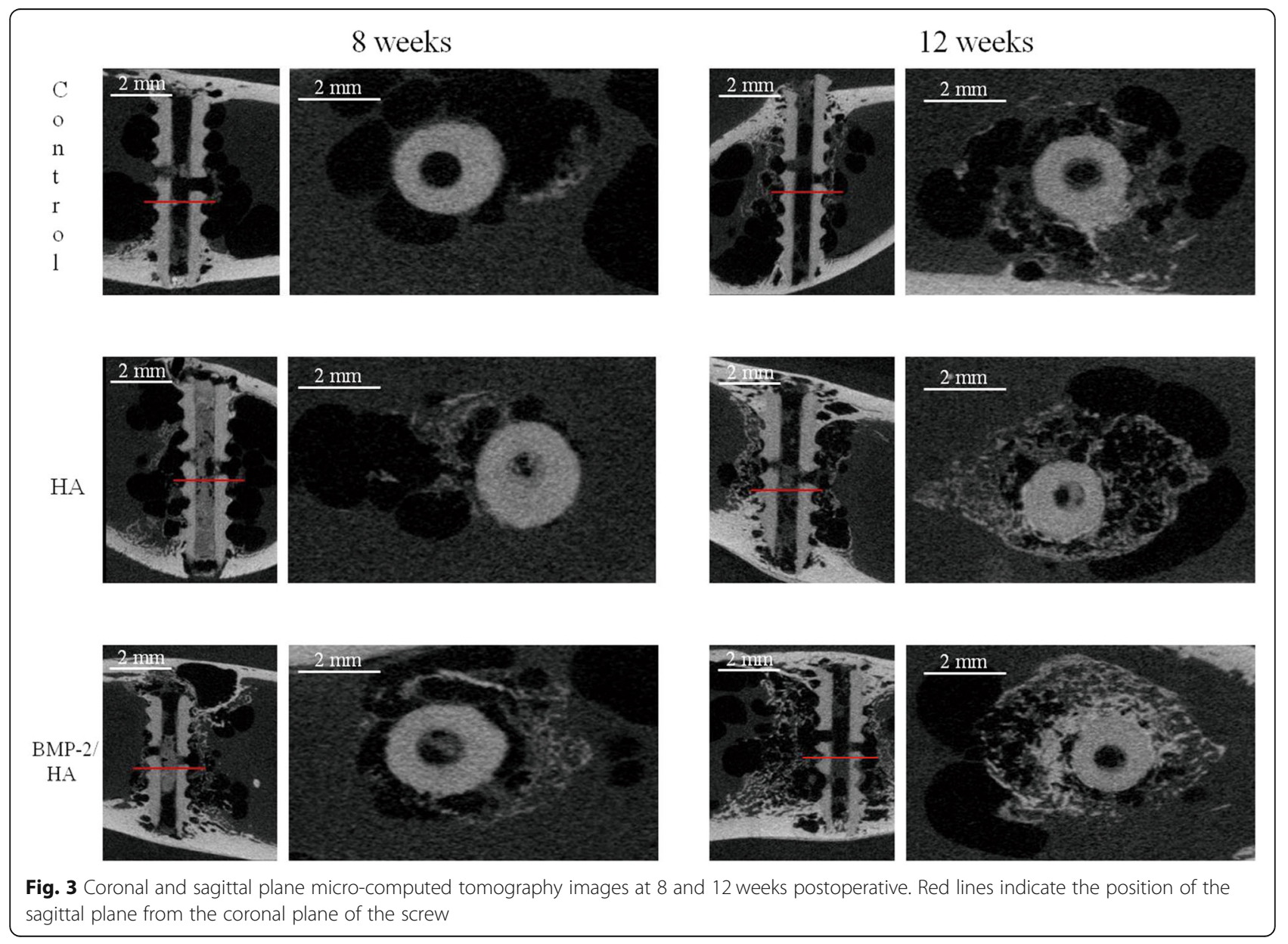



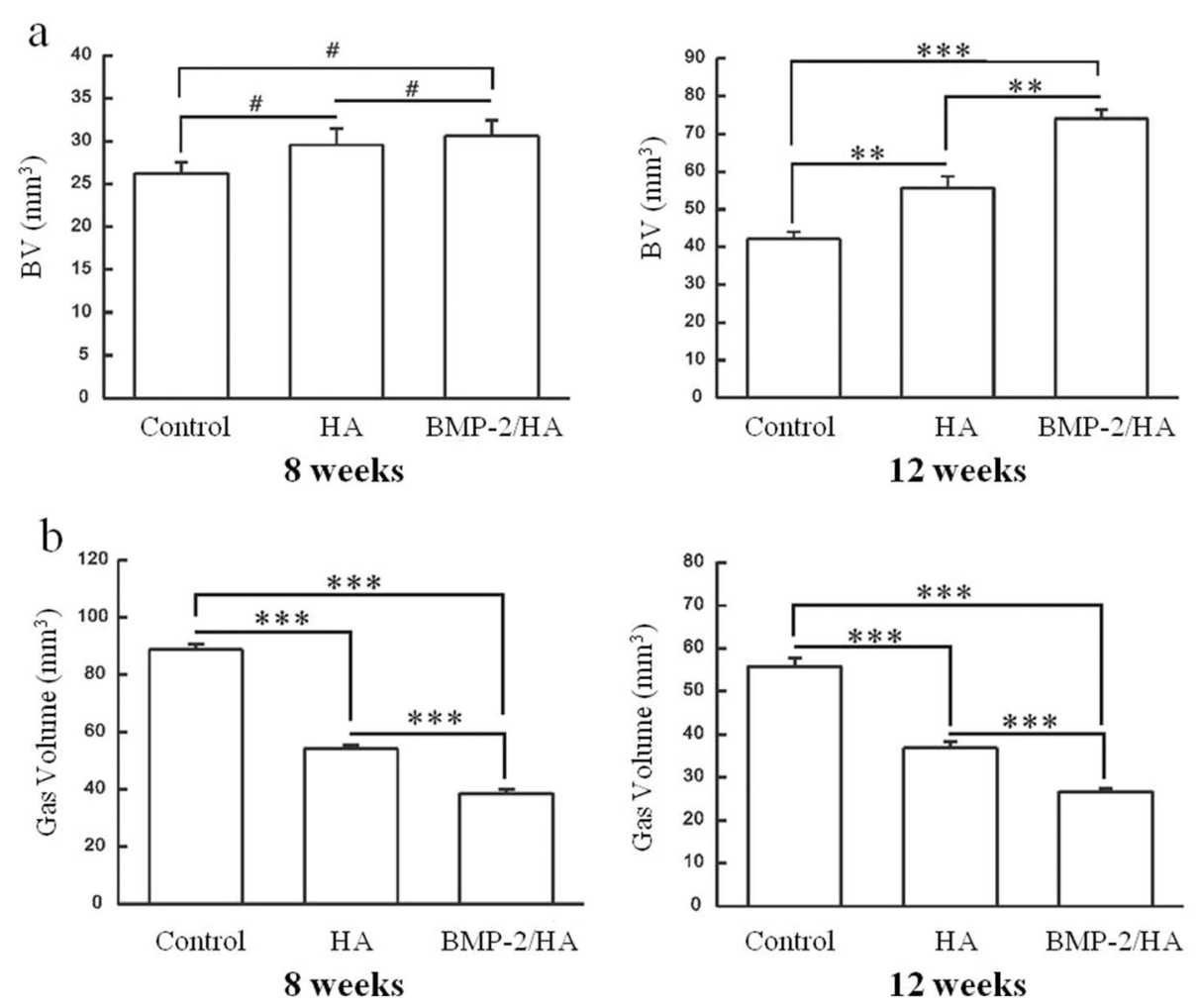

Fig. 4 Quantitative analysis of micro-computed tomography. a Parameters of the new bone volume formation around implants in the control, $\mathrm{HA}$, and BMP-2/HA groups at 8 and 12 weeks. $\mathbf{b}$ Parameters of the gas volume formation around implants in the control, HA, and BMP-2/HA groups at 8 and 12 weeks. Two asterisks indicate significant differences with $p<0.01$, three asterisks indicate significant differences with $p<0.001$, and number sign denotes no significant differences with $p>0.05$

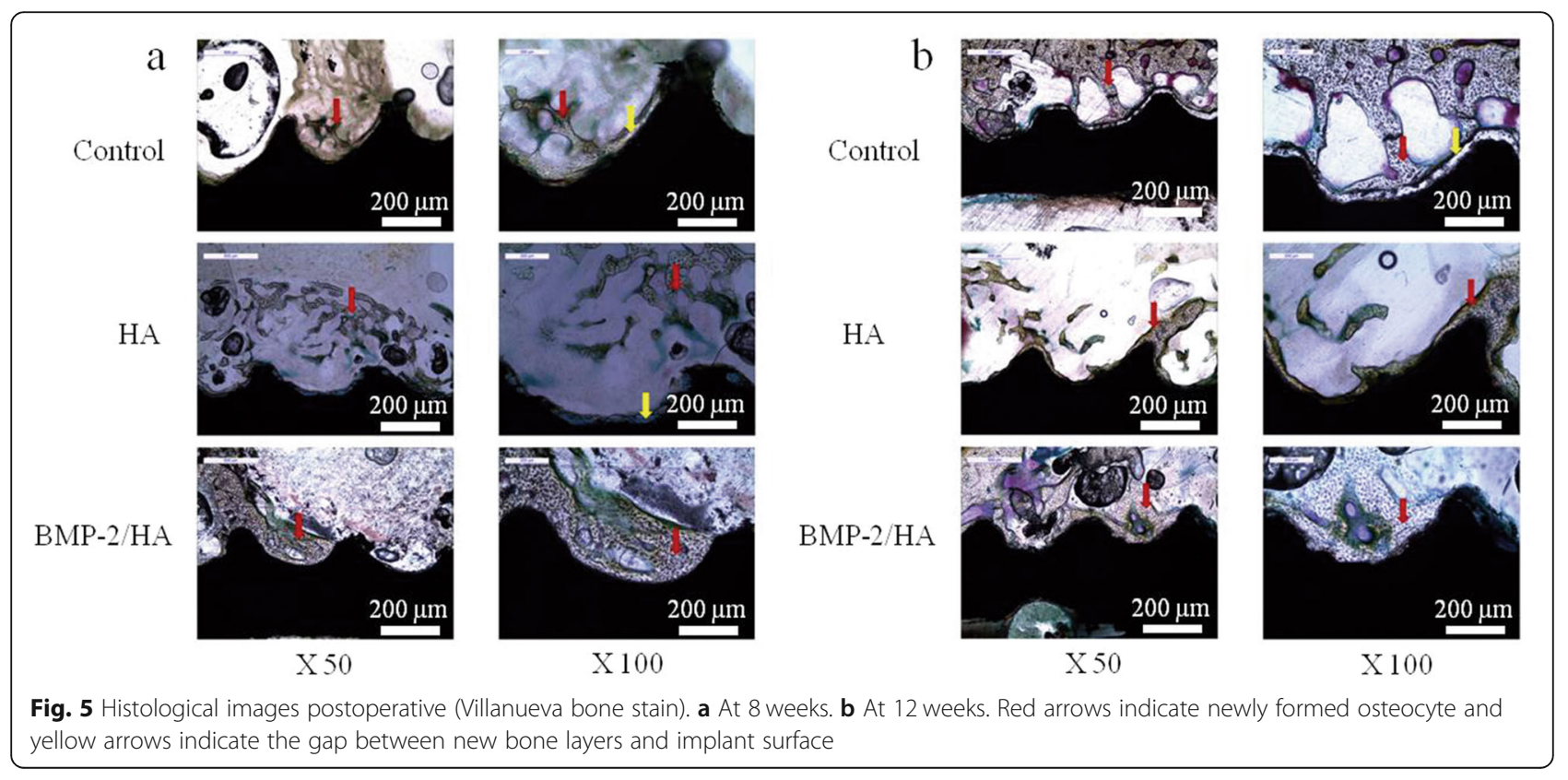




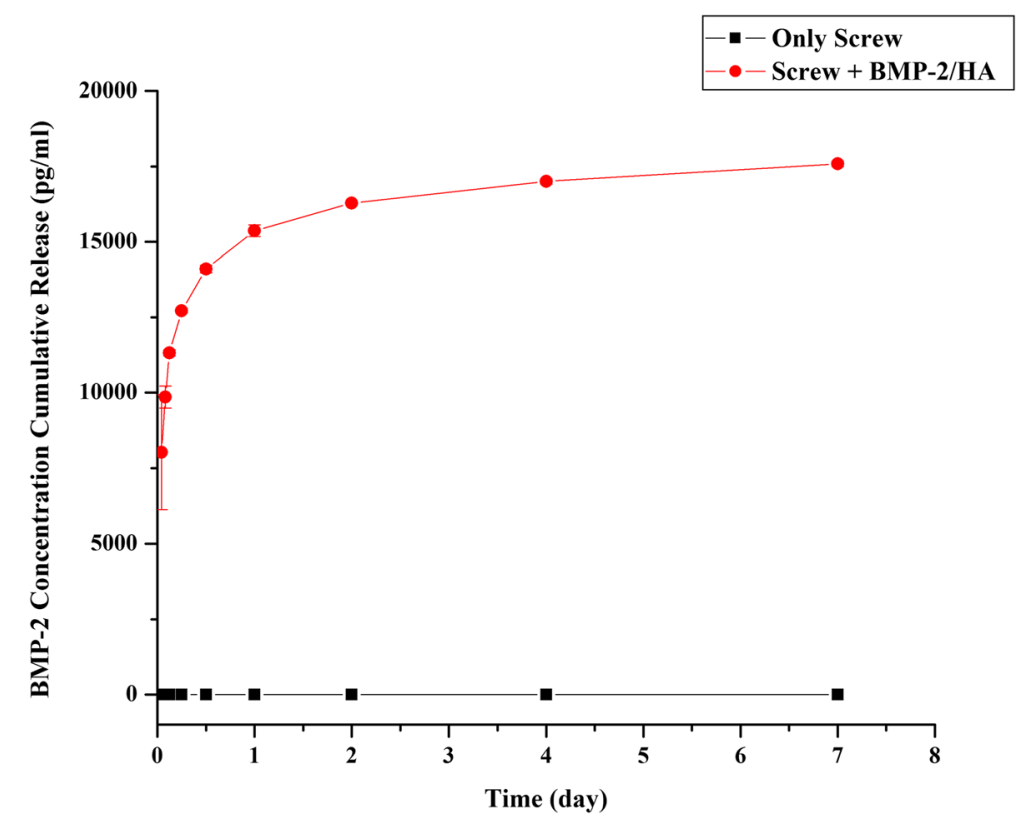

Fig. 6 BMP-2 released profile of the screw treatment with a composite BMP-2/HA compared with the screw with no treatment

was $17,594.97 \pm 90.3132 \mathrm{pg} / \mathrm{ml}$, while in the screw with no treatment was $0 \mathrm{pg} / \mathrm{ml}$ at each tested time point.

\section{Discussion}

The complications of hydrogen gas formed from Mg alloy screws remain controversial. Most research has been within preclinical studies which report gas formation during degradation without specific complications $[8,16]$. In contrast, according to Noviana et al., the authors showed the adverse effect of excessive hydrogen gas evolution to the survival rate of rats implanted with magnesium implants; the persistent presence of gas cavity causes prolonged discomfort and disturbs the balance of blood cell parameters which in turn decreases the survival rate [17]. On bone healing, only a few preclinical and clinical studies have shown the side effects of hydrogen gas production. A hypothesized explanation is that the space-consuming gas pockets raise the inner mechanical pressure, which then inhibits the initial cortical bone healing process. These gas bubbles also potentially decrease osteocyte integration with the implant surface. Therefore, some authors do not recommend $\mathrm{Mg}$ alloy screws for clinical use $[6,7,18]$. We hypothesized that a Mg cannulated screw delivery system with BMP-2/HA would decrease the limitations of $\mathrm{Mg}$ screws relative to hydrogen gas formation.

According to radiographic evaluation, gas formation was first observed at 1 week postoperatively and continued to increase until 8 weeks; after that, the radiolucent area decreased. This result could be explained as gradual new bone formation which replaced the gas space around screws. Another possible explanation is the gas bubble was absorbed into the surrounding tissues [19]. Several studies describe this gas formation through implant degradation which develops in the first week and then decreased over time $[8,11]$. In an in vivo study, $\mathrm{Li}$ et al. observed gas shadows in the soft tissue and bone marrow cavity around $\mathrm{Mg}$ alloy implants during the early implantation period which disappeared 2 months after implantation [20]. However, in our study, the micro-CT scans revealed the gas bubble still remained until 12 weeks (Fig. 3). This could be explained through the implant's local environment and available blood flow, which differentially clear the gas from the implant site. In the present study, the implants were placed in a bone marrow environment, which has a lower water content and blood flow than muscle or subcutaneous tissues thus resulting in potentially slower absorption.

The micro-CT images, at 8 and 12 weeks postoperative, found new bone formation around the implants which replaced the gas bubble space. However, the properties of that circle of bone formed were different between the three groups. Within the control group, the newly formed bone's density appeared to be higher in the peripheral versus the central portion, but in contrast, the other two groups showed opposite results (Fig. 3). This likely relates to the material within the canal of the screw (HA or BMP-2/HA) which is released to the environment around the screw through the cannulated holes and the holes that were placed into the body of the screw. Furthermore, the new bone volume at 12 weeks was significantly higher in the BMP-2/HA group than that in the HA and control groups. At 8 weeks, the bone volume was also higher in 
the BMP-2/HA group than the other groups but not at a statistically significant level at that time (Fig. 4a). In addition, the volume of gas formed around the screw according to micro-CT analysis showed a significantly less amount from the BMP-2/HA group to the HA and control groups at both 8 and 12 weeks postoperatively (Fig. 4b). This shows the potential correlation between the increase in new bone formation and the associated decrease in gas volume.

In addition, at 8 weeks, histological images revealed an improved integration between the newly formed bone and the implant surface within the BMP-2/HA group. The HA group showed a gap between the bone-implant surface while the control group's bone-implant gap was larger and new bone formation was scattered (Fig. 5a). At 12 weeks, new bone formation improved in the three groups, but the control group's gap remained. A gap was not observed in the HA group; however, the bone/implant interface was thinner and more scattered when compared with the BMP-2/HA group (Fig. 5b).

The effect of BMP-2 on bone regeneration has been established in both preclinical studies and clinical trials [21]. According to this study's promising results from radiographs, micro-CT, and histological analysis, the BMP-2/HA delivery system shows remarkable efficiency in forming new bone around the implant when compared with other groups. BMP-2 has proved to be one possible strategy for inducing bone formation, but the successful application of BMP-2 depends on the optimal therapeutic dosage, delivery system, and local circumstances for bone repair; these factors are still under examination [21]. The BMP-2 dose must be sufficient for adequate bone formation, yet not cause the known complications of ectopic ossification, inflammatory reaction, and pain [22]. This inflammation with abnormal bone formation has been observed in rats after 2 weeks in critical-sized femoral bone defects treated with high doses of BMP-2 $(>150 \mu \mathrm{g} / \mathrm{ml})$ [23]. Therefore, in our study, we used an average $40 \mu \mathrm{g} /$ $\mathrm{mL}$ dose of BMP-2 based on our previous research [24, 25] and the baseline dose used in human spinal fusion. No side effects such as skin infection, necrosis, and local inflammation were observed for up to 10 days, which means that acute complications relative to wound site do not occur; besides that, we checked the surgical position on the animal each time we fed it during the period of postoperative to sacrificed animals to harvest the samples as well as during the sample harvesting operation and we did not observe any evidences of local infections or inflammations as well as ectopic bone.

The carrier also plays an important role in the effectiveness of BMP-2. The carriers should be easily sterilized, should be biodegradable, and should have no immunogenicity. They should also provide an osteoconductive matrix that stabilizes the release of BMPs and thereby lowers the required dose [26]. We choose HA as the BMP-2 carrier since it is capable of directly bonding to bone and can improve corrosion resistance as well as restrain the $\mathrm{pH}$ increase. BMP-2 adsorption onto HA can also enhance interfacial strength and improve contact between the HA and surrounding bone. This promotes greater bone regeneration around the screw than HA alone [27]. This study had similar new bone volume formation as other investigations using the same HA carrier for BMP-2 [15, 28, 29].

Screws used in our study are cannulated screws; interestingly, cannulated screws are commonly performed as minimally invasive techniques for the treatment of articular and periarticular fractures. Especially when formal open reduction and internal fixation is contraindicated because of associated soft-tissue compromise and swelling, they can be inserted by way of limited open or percutaneous techniques in many cases to protect the skin and healing. Accurate fracture reduction can be attained through insertion of cannulated screws over a guide pin, resulting in provisional stability of the fracture and reduction of the chance of angulation error [30].

Cannulated headless screws made of magnesium alloy have been available on the market for osteosynthesis; since 2015 in Korea, the $\mathrm{Mg}-5 \mathrm{Ca}-1 \mathrm{Zn}$ alloy screws are commercialized by U\&I Corp. (Gyeonggi-do, Korea), and they are now available in the market as K-MET bioresorbable bone screws, and in Germany, since 2017, the magnesium cannulated screws (alloy MgYREZr) are now commercialized by Syntellix (Hanover, Germany) under the name Magnezix [31]. Plaass et al. reported a clinical study, wherein the $\mathrm{Mg}$ $\mathrm{Y}-\mathrm{RE}-\mathrm{Zr}$ screw was compared with the standard titanium screw for fixation of a modified distal metatarsal osteotomy in 26 patients with a symptomatic hallux valgus [32]. Lee et al. reported the results of a long-term clinical study of $\mathrm{Mg}-$ $5 \mathrm{Ca}-1 \mathrm{Zn}$ alloy screws in 53 distal radius fracture fixation cases [19]. Moreover, some clinical case reports were published to introduce the application of magnesium cannulated screw in clinical. Both authors, Baver et al. and Biber et al., performed magnesium cannulated screws to lateral malleolar fixation. Similarly, Meier et al. used magnesium cannulated screws for scaphoid fracture and Leonhardt et al. used it for fixation of fractures of the condylar head of the mandible $[7$, 33-35]. Despite magnesium cannulated screws have been available on the orthopedic field, complications relative to gas formation around implant have been reported. On the clinical study of Meier et al., they reported about extensive bone cyst and the long time period for bone healing when they use magnesium cannulated screws for scaphoid fracture and they concluded that cannulated magnesium screw is currently not recommended for clinical use in scaphoid fracture [7].

Moreover, the current literature reveals that a wide range of coating materials and coating formation 
techniques are employed to enhance the corrosion resistance of Mg-based alloys. However, most of the in vivo or in vitro studies in the literature investigated the corrosion behavior of the coated samples over a period of maximum of a few days or weeks. The results from the studies may not be used to determine the long-term corrosion performance of the same sample; as the coatings will disappear with time, the corrosion process will accelerate and complications related to gas formation around implant will occur [36]. Even with good coating methods, it cannot completely reduce corrosion rate, which means gas formation around screw still was observed; thus, most coatings for $\mathrm{Mg}$ alloys described in the literature have not been developed for medical applications, and no commercial products are available in the biomedical devices sector [37].

According to the ability to release BMP-2 of the screw evaluation, after screw within composite BMP-2/HA immersed in the SBF, the BMP-2 concentration was found and continued to increase over time (Fig. 6). These results verify that the BMP-2 within the canal of the screw is released to the environment around the screw through the cannulated holes and the holes that were placed into the body of the screw. Therefore, besides the advantages of conventional cannulated screw that we mentioned above, the screws used in our study have special design; two holes were placed in the body of the screw, which connected the cannulated portion to the local environment and enabled slow elution of materials from this section in parallel with the formation of gas around the screw processing; and the material in this case is BMP-2, which was demonstrated with powerful osteoinductive and inducing bone formation ability. Thus, the combination of special design in our cannulated screw and BMP-2 is possible to overcome the complications related to gas formation around magnesium screws.

There are several limitations to our study. First, this study lacked in vitro investigation to examine the interaction between BMP-2 and Mg to determine the effect of BMP-2 on Mg corrosion behavior and biomechanical properties. Instead, this study placed a priority on imaging and histology to observe and quantify the newly formed bone around $\mathrm{Mg}$ implants. Another limitation relates to the singular BMP-2 dose used in this study, which did not compare the effect of different BMP-2 doses on bone formation such as fracture healing, bone defect, or distraction osteogenesis [28, 38, 39]. Future work is required to determine the most ideal BMP-2 dose and other carriers, such as absorbable collagen, $\beta$-tricalcium phosphate, and gelatin.

\section{Conclusions}

The placement of BMP-2/HA within a cannulated $\mathrm{Mg}$ screw enhanced the bone formation ability which replaced the gas void around the implants. Thereby, this combination has the potential to limit the complications of hydrogen gas accumulation. Further research is required to analyze BMP-2 doses and the in vitro interactions between $\mathrm{BMP}-2 / \mathrm{HA}$ and $\mathrm{Mg}$ to include biomechanical evaluations compared to other systems.

\section{Abbreviations}

BMP: Bone morphogenetic protein; HA: Hydroxyapatite; Mg: Magnesium; ROI: Region of interest; VOI: Volume of interest; $\mu \mathrm{CT}$ : Micro-computed tomography

\section{Acknowledgements}

Not applicable.

\section{Funding}

This study was supported by a grant from the CNUH-BRI (Biomedical Research Institute of Chonbuk National University Hospital, CNUH-BRI-2012-02-005).

Availability of data and materials

The datasets supporting the conclusions of this article are included within the article.

\section{Authors' contributions}

LHND wrote the paper. KB contributed to revise the manuscript. SYK and YKK performed the experiments. LHND and KJL analyzed the data. MHL contributed reagents/materials/analysis tools. KBL conceived and designed the experiments. All authors read and approved the final paper.

\section{Ethics approval and consent to participate}

The selection of experimental animals, their management, and the surgical protocols were authorized by the Institutional Animal Care and Use Committee of the Chonbuk National University Laboratory Animal Center, Jeonju, South Korea (approved number: CBNU 2017-0013).

\section{Consent for publication}

Not applicable.

\section{Competing interests}

The authors declare that they have no competing interests.

\section{Publisher's Note}

Springer Nature remains neutral with regard to jurisdictional claims in published maps and institutional affiliations.

\section{Author details}

'Department of Orthopedic Surgery, Chonbuk National University Medical School, Research Institute of Clinical Medicine of Chonbuk National University-Biomedical Research Institute of Chonbuk National University Hospital, Jeonju, South Korea. ${ }^{2}$ Department of Dental Biomaterials and Institute of Biodegradable Materials, Institute of Oral Bioscience and BK 21 Plus project, School of Dentistry, Chonbuk National University, Jeonju, South Korea.

Received: 14 November 2018 Accepted: 4 April 2019

Published online: 29 April 2019

\section{References}

1. Staiger MP, Pietak AM, Huadmai J, Dias G. Magnesium and its alloys as orthopedic biomaterials: a review. Biomaterials. 2006;27(9):1728-34.

2. Okuma T. Magnesium and bone strength. Nutrition. 2001;17(7):679-80.

3. Gu X, Zheng Y, Cheng Y, Zhong S, Xi T. In vitro corrosion and biocompatibility of binary magnesium alloys. Biomaterials. 2009;30(4):484-98.

4. Zhang S, Zhang X, Zhao C, Li J, Song Y, Xie C, et al. Research on an Mg-Zn alloy as a degradable biomaterial. Acta Biomater. 2010;6(2):626-40.

5. Witte F, Hort N, Vogt C, Cohen S, Kainer KU, Willumeit R, et al. Degradable biomaterials based on magnesium corrosion. Curr Opinion Solid State Mater Sci. 2008;12(5):63-72. 
6. Plaass C, Ettinger S, Sonnow L, Koenneker S, Noll Y, Weizbauer A, et al. Early results using a biodegradable magnesium screw for modified chevron osteotomies. J Orthop Res. 2016;34(12):2207-14.

7. Meier R, Panzica M. First results with a resorbable MgYREZr compression screw in unstable scaphoid fractures show extensive bone cysts. Handchir Mikrochir Plast Chir. 2017:49(1):37-41.

8. Kim YK, Lee KB, Kim SY, Bode K, Jang YS, Kwon TY, et al. Gas formation and biological effects of biodegradable magnesium in a preclinical and clinical observation. Sci Technol Adv Mater. 2018;19(1):324-35.

9. Kaesel V, Tai PT, Bach FW, Haferkamp H, Witte F, Windhagen H. Approach to control the corrosion of magnesium by alloying. In: Magnesium: Wiley-VCH Verlag GmbH \& Co. KGaA; 2005. p. 534-9.

10. Gray J, Luan B. Protective coatings on magnesium and its alloys-a critical review. J Alloys Compd. 2002;336(1):88-113.

11. Schaller B, Saulacic N, Imwinkelried T, Beck S, Liu EWY, Gralla J, et al. In vivo degradation of magnesium plate/screw osteosynthesis implant systems: soft and hard tissue response in a calvarial model in miniature pigs. J Craniomaxillofac Surg. 2016;44(3):309-17.

12. Hallman M, Thor A. Bone substitutes and growth factors as an alternative/ complement to autogenous bone for grafting in implant dentistry. Periodontol. 2008:47:172-92.

13. Xiao C, Zhou H, Liu G, Zhang P, Fu Y, Gu P, et al. Bone marrow stromal cells with a combined expression of BMP-2 and VEGF-165 enhanced bone regeneration. Biomed Mater (Bristol, England). 2011;6(1):015013.

14. Annis P, Brodke DS, Spiker WR, Daubs MD, Lawrence BD. The fate of L5-S1 with low-dose BMP-2 and pelvic fixation, with or without interbody fusion, in adult deformity surgery. Spine. 2015;40(11):E634-9.

15. Chung C-H, Kim Y-K, Lee J-S, Jung U-W, Pang E-K, Choi S-H. Rapid bone regeneration by Escherichia coli-derived recombinant human bone morphogenetic protein-2 loaded on a hydroxyapatite carrier in the rabbit calvarial defect model. Biomater Res. 2015;19:17.

16. Song $\mathrm{G}$. Recent progress in corrosion and protection of magnesium alloys. Adv Eng Mater. 2005;7(7):563-86.

17. Noviana D, Paramitha D, Ulum MF, Hermawan $H$. The effect of hydrogen gas evolution of magnesium implant on the postimplantation mortality of rats. J Orthop Transl. 2016;5:9-15.

18. Kraus T, Fischerauer SF, Hänzi AC, Uggowitzer PJ, Löffler JF, Weinberg AM. Magnesium alloys for temporary implants in osteosynthesis: in vivo studies of their degradation and interaction with bone. Acta Biomater. 2012;8(3):1230-8.

19. Lee J-W, Han H-S, Han K-J, Park J, Jeon H, Ok M-R, et al. Long-term clinical study and multiscale analysis of in vivo biodegradation mechanism of $\mathrm{Mg}$ alloy. Proc Natl Acad Sci U S A. 2016;113(3):716-21.

20. Li Z, Gu X, Lou S, Zheng Y. The development of binary Mg-Ca alloys for use as biodegradable materials within bone. Biomaterials. 2008;29(10):1329-44.

21. Termaat MF, Den Boer FC, Bakker FC, Patka P, Haarman HJ. Bone morphogenetic proteins. Development and clinical efficacy in the treatment of fractures and bone defects. J Bone Joint Surg Am. 2005;87(6):1367-78.

22. Carragee EJ, Hurwitz EL, Weiner BK. A critical review of recombinant human bone morphogenetic protein-2 trials in spinal surgery: emerging safety concerns and lessons learned. Spine J. 2011;11(6):471-91.

23. Zara JN, Siu RK, Zhang X, Shen J, Ngo R, Lee M, et al. High doses of bone morphogenetic protein 2 induce structurally abnormal bone and inflammation in vivo. Tissue Eng A. 2011:17(9-10):1389-99.

24. Lee K-B, Taghavi CE, Song K-J, Sintuu C, Yoo JH, Keorochana G, et al. Inflammatory characteristics of rhBMP-2 in vitro and in an in vivo rodent model. Spine. 2011;36(3):E149-E54.

25. Lee KB, Taghavi CE, Murray SS, Song KJ, Keorochana G, Wang JC. BMP induced inflammation: a comparison of rhBMP-7 and rhBMP-2.J Orthop Res. 2012;30(12):1985-94.

26. Bessa PC, Casal M, Reis RL. Bone morphogenetic proteins in tissue engineering: the road from laboratory to clinic, part II (BMP delivery). J Tissue Eng Regen Med. 2008;2(2-3):81-96.

27. Rohanizadeh $R$, Chung K. Hydroxyapatite as a carrier for bone morphogenetic protein. J Oral Implantol. 2011;37(6):659-72.

28. Notodihardjo FZ, Kakudo N, Kushida S, Suzuki K, Kusumoto K. Bone regeneration with BMP-2 and hydroxyapatite in critical-size calvarial defects in rats. J Cranio-Maxillofac Surg. 2012;40(3):287-91.

29. Jun S-H, Lee E-J, Jang T-S, Kim H-E, Jang J-H, Koh Y-H. Bone morphogenic protein-2 (BMP-2) loaded hybrid coating on porous hydroxyapatite scaffolds for bone tissue engineering. J Mater Sci Mater Med. 2013;24(3):773-82.

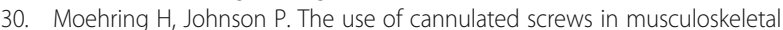
trauma. A review of surgical techniques. Orthop Rev. 1994;23(Suppl):10-21.
31. Hermawan $\mathrm{H}$. Updates on the research and development of absorbable metals for biomedical applications. Prog Biomater. 2018;7(2):93-110.

32. Plaass C, von Falck C, Ettinger S, Sonnow L, Calderone F, Weizbauer A, et al. Bioabsorbable magnesium versus standard titanium compression screws for fixation of distal metatarsal osteotomies-3 year results of a randomized clinical trial. J Orthop Sci. 2018;23(2):321-7.

33. Acar B, Unal M, Turan A, Kose O. Isolated lateral malleolar fracture treated with a bioabsorbable magnesium compression screw. Cureus. 2018;10(4):e2539.

34. Biber R, Pauser J, Brem M, Bail HJ. Bioabsorbable metal screws in traumatology: a promising innovation. Trauma Case Rep. 2017;8:11-5.

35. Leonhardt H, Franke A, McLeod N, Lauer G, Nowak A. Fixation of fractures of the condylar head of the mandible with a new magnesium-alloy biodegradable cannulated headless bone screw. Br J Oral Maxillofac Surg. 2017;55(6):623-5.

36. Uddin M, Hall C, Murphy P. Surface treatments for controlling corrosion rate of biodegradable Mg and Mg-based alloy implants. Sci Technol Adv Mater. 2015;16(5):053501.

37. Hornberger $\mathrm{H}$, Virtanen $\mathrm{S}$, Boccaccini A. Biomedical coatings on magnesium alloys-a review. Acta Biomater. 2012;8(7):2442-55.

38. Sailhan F, Gleyzolle B, Parot R, Guerini H, Viguier E. Rh-BMP-2 in distraction osteogenesis: dose effect and premature consolidation. Injury. 2010;41(7): 680-6.

39. Harada Y, Itoi T, Wakitani S, Irie H, Sakamoto M, Zhao D, et al. Effect of Escherichia coli-produced recombinant human bone morphogenetic protein 2 on the regeneration of canine segmental ulnar defects. J Bone Miner Metab. 2012;30(4):388-99.

\section{Ready to submit your research? Choose BMC and benefit from:}

- fast, convenient online submission

- thorough peer review by experienced researchers in your field

- rapid publication on acceptance

- support for research data, including large and complex data types

- gold Open Access which fosters wider collaboration and increased citations

- maximum visibility for your research: over $100 \mathrm{M}$ website views per year

At $\mathrm{BMC}$, research is always in progress.

Learn more biomedcentral.com/submissions 